\title{
An Overview of Various Template Matching Methodologies in Image Processing
}

\author{
Paridhi Swaroop \\ M.Tech(Computer Science) \\ Banasthali Vidhyapeeth \\ Rajasthan
}

\author{
Neelam Sharma, PhD \\ Asst. Professor \\ Banasthali Vidhyapeeth \\ Rajasthan
}

\begin{abstract}
The recognition and classification of objects in images is a emerging trend within the discipline of computer vision community. A general image processing problem is to decide the vicinity of an object by means of a template once the scale and rotation of the true target are unknown. Template is primarily a sub-part of an object that's to be matched amongst entirely different objects. The techniques of template matching are flexible and generally easy to make use of, that makes it one amongst the most famous strategies of object localization. Template matching is carried out in versatile fields like image processing,signal processing, video compression and pattern recognition.This paper gives brief description of applications and methods where template matching methods were used.
\end{abstract}

\section{General Terms}

Template matching,computer vision,image processing,object recognition.

\section{Keywords}

Template Matching, image processing, object recognition.

\section{INTRODUCTION}

Template Matching may be a high-level machine vision method which determines the components of a figure which matches a predefined template.Template Matching could be a methodology in digital image processing to identify little components of a figure which match a template image.We tend to match a template to an image wherever the template is a sub image which contains the form which we want to find out. This technique is repeated for the whole image, and the point which leads to a best match, the utmost count, is defined to be the point wherever the shape (given by the template) lies inside the image. Templates are usually employed to print characters, identify numbers, and other little, simple objects. It can be used for detection of edges in figures in manufacturing as a part of quality control and a means to navigate a mobile robot. Template Matching is a strategy for discovering zones of an image which matches (are indistinguishable ) a template image (patch). We require 2 crucial segments.

Source image (I): The picture inside which we are hoping to find out a match to the template image.

Template image (T): The patch image that can be compared to the template image and our objective is to discover the most effective technique for the best matching region. Matching technique not solely takes the similarity measure however it computes the inaccuracy among images reckoning on the difference by means of Mean Squared Error(MSE) metric.
MSE(Mean Squared Error)

$$
=\frac{\sum \sum(\text { Temporary }(\mathrm{u}, \mathrm{v})-\text { Target }(\mathrm{u}, \mathrm{v})}{\text { Number of pixels }}
$$

wherever, Temporary $(\mathrm{u}, \mathrm{v})$ and Target $(\mathrm{u}, \mathrm{v})$ are the strength values of Template image and the input image respectively.

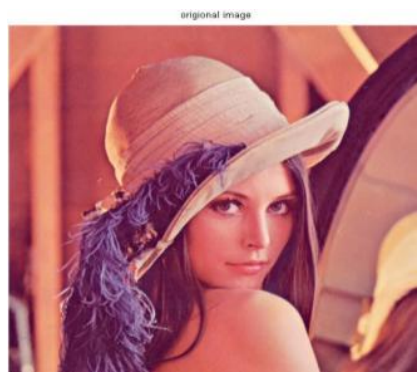

eye template

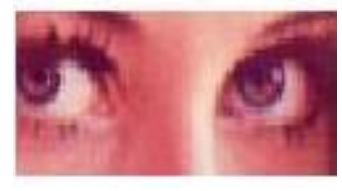

Fig 1: Example of template matching.

An example which describes template matching. This figure describes template based eye detection. The template is correlated with absolutely distinctive regions of a face image.The regions of a face that offers maximum correlation with template refers to the eye region.This approach is effective in both the instances like closed eyes along with open eyes and conveys numerous simulation outcome.

\subsection{Template Matching Approaches}

General categorizations of template or image matching approaches are Featured-based approach and Template or Area based approach.

\subsubsection{Featured-based approach}

The Featured-based method is appropriate while both reference and template images have more connection with regards to features and control points. The features comprises of points, a surface model which needs to be matched,and curves. At this point, the goal is to position the pairwise association amongst reference and layout picture using their spatial relations or descriptors of components.

\subsubsection{Area-based approach}

The Area-based methods are typically referred to as correlation like methods or template matching methods, that is the blend of feature matching, feature detection , motion tracking,occlusion handling etc. Area-based methods merge the matching part with the feature detection step. These techniques manage the pictures without attempting to identify the remarkable article. Windows of predefined size are used for the estimation of correspondence.

\subsubsection{Template-based approach}

Template-based template matching approach could probably require sampling of a huge quantity of points, it's possible to cut back the amount of sampling points via diminshing the 
resolution of the search and template images via the same factor and performs operation on resulting downsized images (multiresolution,or Pyramid (image processing)), providing a search window of data points inside the search image in order that the template doesn't have to be compelled to look for each possible data point and the mixture of the two.

\subsubsection{Motion Tracking and Occlusion Handling}

For the templates which can't provide and may not provide an instantaneous match, in that case Eigen spaces may be used, which provides the details of matching image beneath numerous conditions, appropriate matching poses or colour contrast.For instance, if the person turned into searching out a specimen, the Eigen spaces may include templates of specimen totally different in numerous positions to camera with different lighting conditions or expressions .There is feasibility for the matching figure to be occluded via an associated item or issues involved in movement turn out to be ambiguous. One amongst the probable answers for that can be to separate the template into more than one sub images and carry out matching on them.

\section{METHODOLOGY}

The steps of template matching are described below.It is represented with the help of flow diagram.

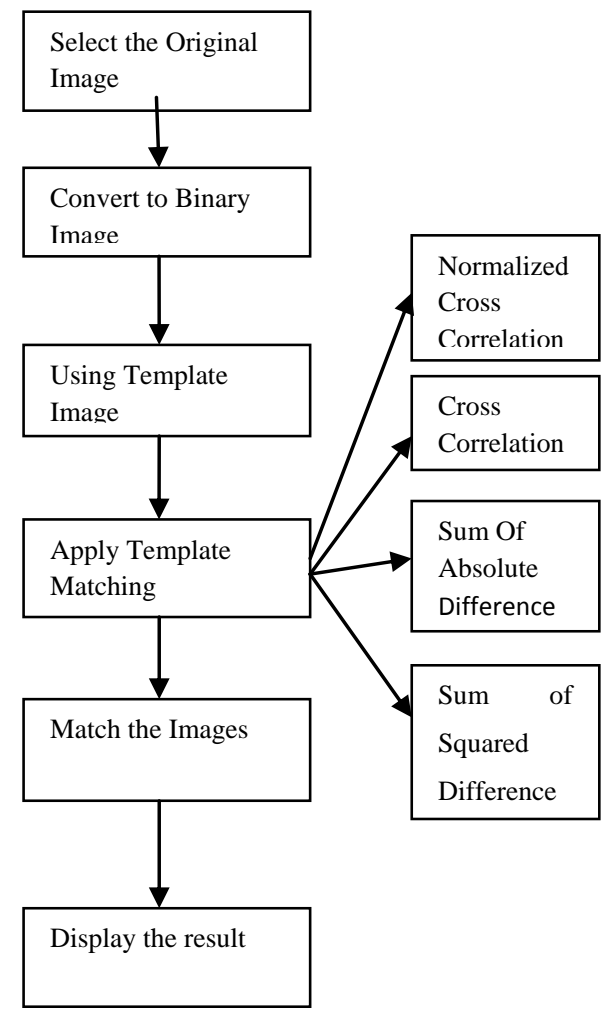

Fig 2:Flow Diagram of Template Matching approach

1. First we select the original image. The image will be in file formats such as JPG/JPEG, PNG etc.

2. Convert to Binary Image. The process to convert the color image into white and black image is named as a binary image. This method is based totally on numerous color transforms.It analyze the values of grey scale and also achieves the grey image according to the R, G, B value within the image.The technique of template matching can be easilycarried out on edge figures or grey figures.
3. Using the template image.Template image may be a small portion of an input image and is used to find the template within the given search image.

4. Apply Template Matching Techniques like Normalized Cross Correlation ,Cross Correlation,Sum of Squared Difference.

5. Then match the images with the original image.

6. Display the result.

\section{TECHNIQUES}

The template matching matching techniques are described below:

\section{Naive Template Matching}

Assume that you are provided a picture of a plug and our goal is to search out its pins. We are supplied with the pattern image that represents the reference object we are looking for and therefore the input image to be used that position the pattern above the image at each attainable location, and every instant we tend to calculate some numeric measure of similarity amid the pattern and therefore the image section it presently overlaps. At last we tend to determine the locations that give the most effective similarity measures as the feasible pattern positions.

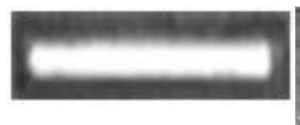

Pattern Image

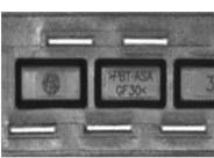

Input Image
Fig 3:Signify the pattern image chosen to be matched inside input image.

\section{Image Correlation Matching}

The problem that occurs in Naive Template Matching is in computing the similarity measure of the aligned pattern image and therefore the overlapped section of the input image, that's equal to computing a similarity measure of 2 figures of same dimensions. So the numeric measure of image similarity is known as image-correlation

\subsection{Cross-Correlation}

Cross-correlation $($ Image 1, Image 2$)=\sum_{\mathrm{u}, \mathrm{v}} \operatorname{Image} 1(\mathrm{u}, \mathrm{v}) \times$ Image2(u, v).

Table 1:It shows the cross correlation of two images.

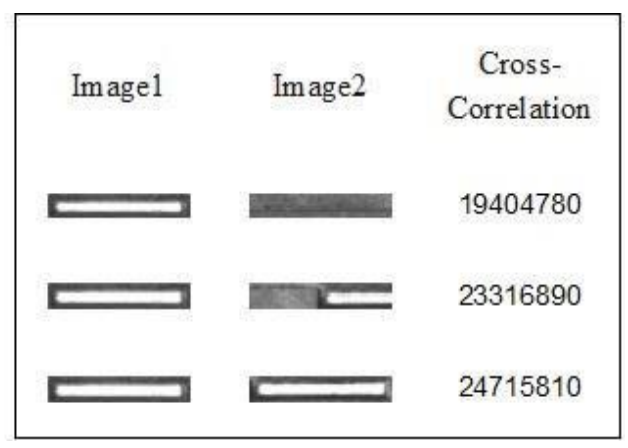

Here we have taken two images Image 1 and Image 2 and their pixel coordinates $\mathrm{u}$ and $\mathrm{v}$.The fundamental strategy of computing the image correlation is so referred to as crosscorrelation, that is basically a simple sum of pair wise multiplications of corresponding pixel values of the images. 

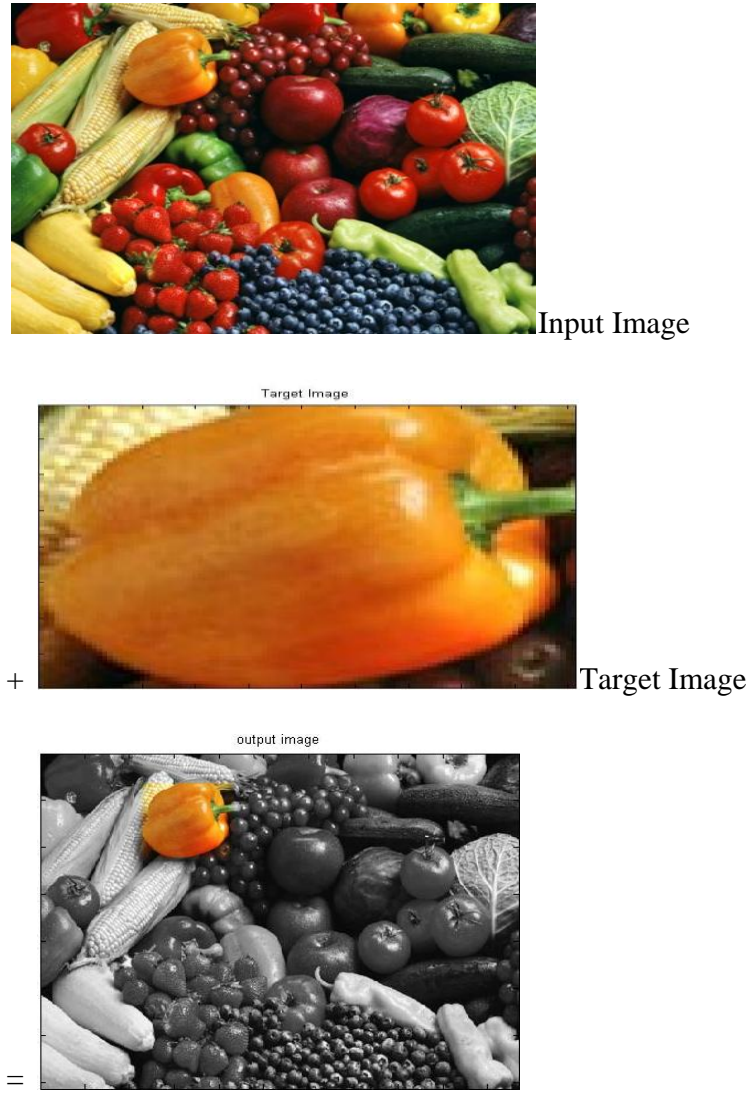

Fig 4:Template Matching with the help of crosscorrelation

\subsection{Normalized cross-correlation}

Normalized cross-correlation is an improved model of the traditional cross-correlation methodology which bring in 2 improvements over the primary one:

- The outcomes are constant to the global brightness changes,that is darkening of whichever figure or consistent brightening have no impact on end result (this can be achieved via subtracting the mean image brightness from every pixel value).

- The final correlation value is scaled to $[-1,1]$ range, in order that NCC of 2 alike figures is equal to 1.0, though NCC of a figure plus its negation is equal to -1.0 .

- TABLE.2:It shows normalized cross correlation of two images

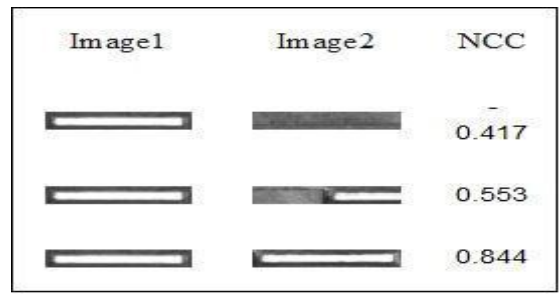

- Formula of NCC is: NCC(Image1,Image2)=

$$
\begin{aligned}
\frac{1}{N \sigma 1 \sigma 2 \sigma} \sum_{u, v}[(\text { Image } & 1(u, v)-\overline{\text { Image } 1}) \\
& \times(\text { Image } 2(u, v)-\overline{\text { Image } 2})]
\end{aligned}
$$

In this formula, we have taken two images Image 1 and Image 2 and their pixel coordinates $\mathrm{u}, \mathrm{v}$ and $\sigma$ is some constant. Normalized cross-correlation can be generally applied as an efficient resemblance measure meant for matching applications.But, conventional correlation-based image matching techniques may not succeed while there are significant scale changes or large rotations among the two figures. That's the reason why normalized cross-correlation is sensitive to revolution in addition to scale changes.

Normalized Cross Correlation is more complicated as compared to both SSD and SAD algorithms as it entails several division, square root operations and multiplication.

NCC technique is used in face recognition system. Normalized Cross Correlation (NCC) is the technique that is employed in image registration for matching the template with an image. On the other hand, NCC was influenced via factors such as illumination and clutter background issues. In case of NCC, there's a big increase within the inaccuracy rate which is because of the shaded input figure. The values of the pixels of the shaded portions are lesser than the portions of a image and this encompasses a high proportion of NCC among template image \& input image that can conclude a incorrect location.

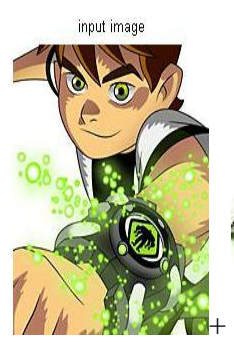

Input Image

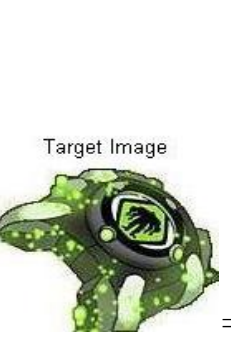

Target Image

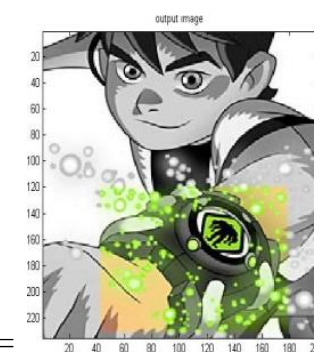

Output Image
Fig 5:Template Matching with the help of Normalised Cross Correlation

\section{Sum of Absolute Difference}

Sum of Absolute Difference can be computed via obtaining the absolute difference among every pixel within the Source image(original block) and therefore the subsequent pixel within the block that's meant for the aim of comparison. It could be used as a measure to determine the similarity among image blocks. It is used in many fields such as object recognition,estimation of movement for video compression and generates dissimilarity maps designed for stereo imagery.The Sum of Absolute Difference(SAD) can be computed as:

$$
\operatorname{SAD}(\mathrm{a}, \mathrm{b})=\sum_{\mathrm{k}=0}^{(\mathrm{M}-1)(\mathrm{N}-1)} \sum_{\mathrm{l}=0}^{(\mathrm{N}-1)}[\mathrm{S}(\mathrm{a}+\mathrm{k}, \mathrm{b}+\mathrm{l})-\mathrm{T}(\mathrm{k}, \mathrm{l})]
$$

The SAD(Sum Of Absolute Difference) is used to measure the similarity among template image $\mathrm{T}$ and sub-images within the source images S.It works via computing the absolute difference among every pixel in template image $\mathrm{T}$ and as well as subsequent pixel within the sub-images which is intended for comparision in the source image S.Then we find the summation of all the differences obtained to produce a straightforward metric of similarity.For instance,suppose a two-dimensional $\mathrm{A} \times \mathrm{B}$ template, $\mathrm{T}(\mathrm{a}, \mathrm{b})$ which is used for matching inside a $S(a, b)$ i.e the source image that has $S \times T$ wherever $(\mathrm{S}>\mathrm{A} \& \mathrm{~T}>\mathrm{B})$. For every pixel at location $(\mathrm{a}, \mathrm{b})$ within the figure,according to the method that we have described below[7] we can calculate the SAD distance. 
1. The difference between 2 points in 1-D is often calculated as follows:

$$
\mathrm{d}(\mathrm{C}, \mathrm{D})=|\mathrm{a} 1-\mathrm{a} 2| \ldots \ldots \ldots \ldots \ldots \ldots
$$

2. The difference among 2 points in $2-\mathrm{D}$ is often calculated as follows.

$$
\mathrm{D}(\mathrm{C}, \mathrm{D})=\sqrt{(a 1-a 2)^{2}+(b 1-b 2)^{2}}
$$

3. The difference among 2 functions $h(x) \& i(x)$ is often calculated as follows.

$$
\mathrm{D}(\mathrm{h}, \mathrm{i})=\int|h(x)-i(x)| d(x)
$$

4. The difference between 2 matrices $\mathrm{C} \& \mathrm{D}$ is often computed as follows.

$$
\mathrm{D}(\mathrm{C}, \mathrm{D})=\mathrm{C}-\mathrm{D} \text {. }
$$

As per the formula obtained above ,the lesser the distance measure acquired via SAD function among the template image $\mathrm{T}$ and a sub-image within the source image $\mathrm{S}$, the closer the match among searched template along with the corresponding sub-picture is.As a end result,if the distance obtained via SAD function is 0 , the neighbourhood sub-image is much like a template.

Then those differences will be summed up to determine the similarity amongst two figures, the subtraction of two matrices will produce a new matrix.For instance.

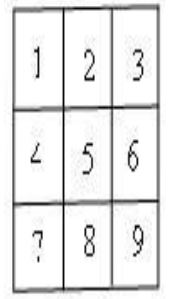

A

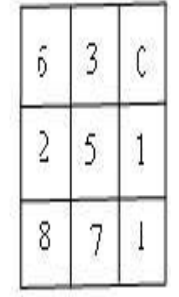

B
Fig 6:Differences between the matrices

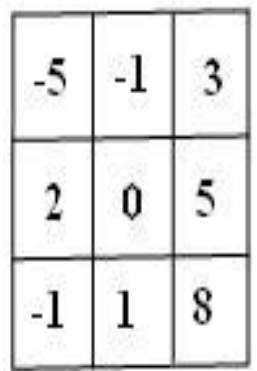

Fig 7:Resulting matrix

Here are some negative values present in the above matrix, hence,we will obtain the absolute value of these matrix elements and after that calculate the sum of these elements. The results that we have obtained after summation povides SAD distance among the image window and template
window.SAD (Sum Of Absolute Difference) is computed via the equation[7].

$\mathrm{D}(\mathrm{C}, \mathrm{D})=\sum_{m} \sum_{n}|A(m, n) B(m, n)|$

$\mathrm{SAD}=5+1+3+2+0+5+1+1+8=26$

During comparison with other general similarity strategies that are correlation-based particularly SSD, NCC,SAD is straightforward, less complex and much more accurate.If 2 image windows have the face then sometimes it is not accurate and that they had almost equal SAD thus to boost this algorithm we want to normalize equation five to search out the optimum image window which contains face.The equation given below explaines $\operatorname{OSAD}$ (Optimized Sum of Absolute Difference)

$\mathrm{d}(\mathrm{C}, \mathrm{D})=\sum_{m} \sum_{n}|C(m, n)-D(m, n)| / \max (\mathrm{C}(\mathrm{m}, \mathrm{n}), \mathrm{D}(\mathrm{m}, \mathrm{n}))$

However, due to the illumination and clutter background issues NCC is affected for the reason that at times there are non-face blocks which had nearly identical value as that of average face template matrix. This drawback is resolved by the use of Sum of Absolute Differences algorithm (SAD) for image compressing and object tracking however, for giving more accurate positions of face within the input image SAD needs more optimization.Moreover,SAD will provide high localization rate for facial image wherever the image is with high illumination variation however variation in background may affect it.

But in case of SSD, the accuracy is acceptable however its complexity is more than OSAD and SAD and so that it maximizes the error rate.

\section{SUM OF SQUARED DIFFERENCE}

Sum of Squared Differences (SSD) is procedure that is employed in image registration for matching the template with an image. Moreover, it also test the effect of template image on output image when there is noise and rotation. The measure of variation or deviation from the mean is represented by sum of squares.It is calculated as a summations of the squares of the variations from the mean. The performance of this method is done by making the comparison based on the value of correlation coefficient and that produced from different template images.It can be computed via formula described below.

$$
c(a, b)=\sum_{\mathrm{k}=-\mathrm{W}}^{\mathrm{W}} \sum_{\mathrm{l}=-\mathrm{H}}^{\mathrm{H}}[\mathrm{f}(\mathrm{a}+\mathrm{b}, \mathrm{b}+\mathrm{e})-\mathrm{t}(\mathrm{k}, \mathrm{e})]^{2}
$$

This measure includes a higher computational complexity as compared to SAD algorithmic rule as a result of it involves varied multiplication operations.It is commonly known as block matching approach.SSD is usually used owing to its simplicity and comparatively low computational price,generally we tend to get better results using Normalized Cross Correlation. For the purpose of matching images it's important that SSD should be less. 

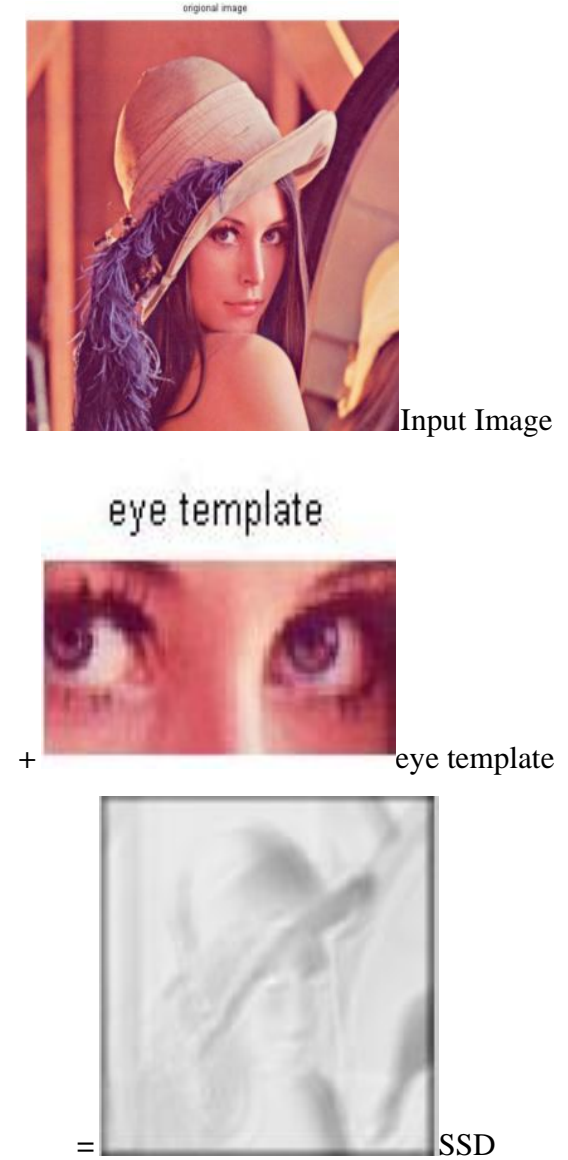

Fig 8:Sum of Squared Difference using template matching

\section{DRAWBACKS OF THE ABOVE METHODS}

There are the following drawbacks of the above technologies . The pattern occurrences are compelled to conserve the orientation of the reference image pattern. These methods are inefficient as it is time-consuming in computing the pattern correlation image for images varying from medium to large images.So some new technologies have been developed.

\section{ADVANCED METHODS}

There are 2 methods:

\section{Grayscale based Matching}

It is an enhanced Template Matching method which is an extension of correlation-based pattern detection,enhances its efficiency and allows to search out for pattern occurrences not considering its orientation.It is done via not simply computing the pattern image pyramid one for every attainable rotation of the pattern. There are many applications of grayscale matching such as computer animation, humancomputer interaction and virtual reality to human motion analysis.This can be done via calculating not just single pattern image pyramid.This algorithm make out the pairs (pattern position, pattern orientation) throughout the pyramid search of the input image instead of sole pattern position. The approach of pyramid matching used together by means of multi-angle search is referred to as Grayscale-based TMatching method.

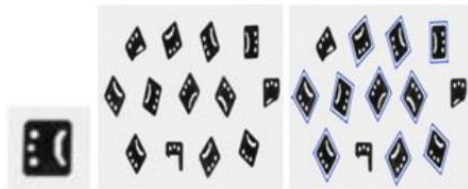

Fig 9:(1)Pattern Image (2)Input Image (3)Results of multiangle matching.

\section{Edge based matching}

Edge-based Matching improves this methodology even a lot via off-putting the computation to the object edge-areas Edgebased Matching updrades the formerly explained Gray-scalebased Matching via the use of 1 vital statement - that the form of any object is outlined only by the form of its edges. Thus rather than matching the entire pattern, we tend to take out its edges and then match solely the close by pixels, therefore keeping away from some superfluous computations.Normally in these applications the acquired speed-up is typically important.

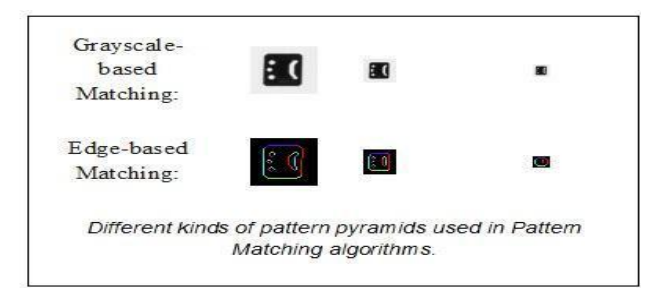

Fig 10:describes various pattern mathing methods

\section{APPLICATION AREAS OF TEMPLATE MATCHING}

Following are the application areas of template matching:

\section{Object Recognition using Template Matching}

Object recognition is job of discovering a known item inside an image or video sequence. It's(object recognition) used to properly determine objects in a scene and estimate their cause (location and orientation).The purpose is to understand the capability of existing object recognition methods to search out alike objects once input is completely of image type. We' $d$ like to rearrange these objects which are visible to us. These objects are totally visible or partly hidden behind another object. Similar objects might also be available in the various pose. The identification of those objects is easy for human being as he can easily identify any object based on his knowledge or expertise yet it is much hard to distinguish a specific item for a machine. The machine has to learn how to recognize any object. For this issue, certain algorithms are proposed. With the assistance of those algorithms, a machine will understand objects present in the various pose, lightning conditions, camera parameters, appearance etc. For instance, the writing style of various individuals is totally different Two person can compose one letter with varied designs .

\section{Biological area}

It is used in biological science such as Nuclear Agriculture and Molecular Biology.It involves applications that involve the use of camera-based hardware systems or colored scanners for inputting pictures.The software package that's has been designed for such purpose is the BIAS software that supports DOS and Windows friendly Color-Pro software which is developed in Electronics Systems Division and Comprehensive Image method. It has the following features like color image analysis for evaluation of leaf ,chlorophyll and defected leaf area. For plant breeding estimation of leaves area was extremely necessary. In olden days, leaf area meters are used for this function. However nowadays image analysis 
is used for measurement of leaf area. The image of leaf is initially taken via camera or a scanner and so analyzed via the Color Pro software package designed via Electronics Systems Division. A range of color plates as well as chlorophyll meters was earlier employed to examine chlorophyll substance of leaf inside situ.

\section{Eye Detection in a Facial Image}

In this technique, we are provided with an eye template and a face image. Then we find the correlation of an eye template through the overlapping areas of the face image ,the section that offers the maximum correlation coefficient with the eye template is referred to as eye region, this is how eye image is found out.

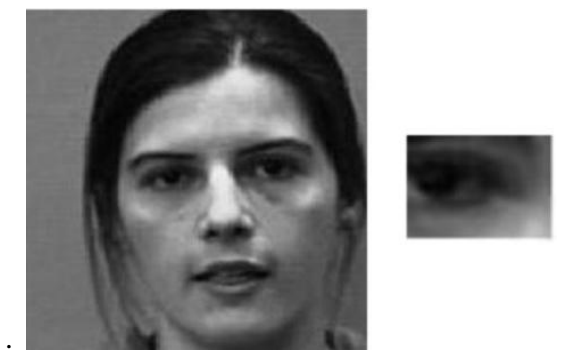

Fig 11 :shows eye template

Assume that $\mathrm{I}$ is an image of size $a \times \mathrm{b} \& \mathrm{~T}$ is other image that has size $s \times \mathrm{t}$. Template matching is defined as a search methodology that locate the portion within $I$ of dimensions $s \times$ $t$ wherever $T$ has the highest cross-correlation coefficient related to it.

\section{The Algorithm:}

The methodology of template matching is explained with the help an algorithm, that is straightforward and simple to execute. The rules of an algorithm are described below:

- Suppose we take an eye template that has size $a \times b$.

- The normalized two-dimensional auto-correlation of an eye template is determined.

- The normalized two-dimensional cross-correlation of an eye template with numerous overlapping sections of a face image are found out.

- The mean squared error (MSE) of auto correlation as well as cross-correlation of various areas are find out. The value that gives the minimum MSE is determined as well as stored.

- The sections of a face that has lowest MSE signify eye region.Matching method not solely obtains similarity measure however it computes the inaccuracy among images reckoning on its difference by means of Mean Squared Error.

\section{Remote Sensing}

Remote Sensing may be applied at precise wavelengths at the same time giving thousands of digital images. Its knowledge can be gathered from a hyperspectral devices contains not solely the visible spectrum however it contains both ultraviolet and infrared ranges. It's general to list the hyperspectral data in a 3-D array or "cube", with the first 2-D matching to geographical dimensions and therefore the third one similar to the spectrum. During hyper spectral categorization and particularly target detection, the most important purpose was to seek out spatial pixels in 3-D hyperspectral cube data for a few best known spectral signals of interest. Though,it becomes complicated since there is variability and uncertainty of every material's spectral signature. These difficulties comprises of noise from atmospherical conditions, illumination, location and sensor control etc, all of that rely on once and wherever the image was taken.

\section{LIMITATION}

Following are the limitations of template matching:

- Templates are not rotation or scale invariant.

- Slight change in size or orientation variations can cause problems.

- It often use several templates to represent one object.

- Templates may be of different sizes.

- Rotations of the same template.

- Particularly if you search the entire image or if you use several templates in that case template matching is a very expensive operation.

- Template matching is easily "parallelized".

- Template Matching requires high computational power because the detection of large patterns of an image is very time taking.

\section{ADVANTAGE}

Estimate become quite good with enough data..Template matching is the most efficient technique to be used in pattern recognition machines which read numbers and letters that are available in standardized, constrained contexts (means scanners which reads your financial credit number from machines, checks that read postal zip codes off from envelopes).

\section{ENHANCING THE ACCURACY OF TEMPLATE MATCHING}

Advancements may be made to the matching methodology via the use of multiple template, the additional templates that we take may have rotations and dissimilar scales.It's also achievable to boost the accuracy of the matching technique via combining feature-based along with template-based methodology. This needs that the search and template images had characteristics which are understandable enough so that it can supports feature matching.

\section{CONCLUSION}

Template matching encompasses a wide scope in enormous devastating regions. This paper defines various effective techniques that have been by now executed and has excellent application rate in several fields with their consequences that assist writers in getting a summary of different template matching algorithm and its relevances.

\section{FUTURE SCOPE}

Proposed system is just supposed to seek out specific shape from certain image, if it comes to relate with color the proposed system won't work. For image with several edges, edge detector rule would possibly takes longer time to make binary image, this reflects on total time taken, and system won't offer end product in desired time.In upcoming time first step will be to implement proposed system on real world scenario with the help of graphics boards. Afterwards if required, noisy images may be handled, as effects which 
shows that, with the increase of noise, the efficiency of system decreases.

\section{ACKNOWLEDGEMENT}

My special thanks to my guide Dr.Neelam Sharma for taking me under her supervision encouraging me to enter into the domain of Image Processing for giving me this wonderful opportunity to conduct my study in this area with her constant support,help and interaction.I have also taken help of research paper which has helped me a lot in development of my paper.

\section{REFERENCES}

[1] Nilamani Bhoi,Mihir Narayan Mohanty. "Template Matching based Eye Detection in Facial Image".International Journal of Computer Applications (0975-8887)Volume 12-No.5,December 2010

[2] T. Mahalakshmi, P. Swaminathan and R. Muthaiah.“ An Overview of Template Matching Technique in Image Processing". Research Journal of Applied Sciences, Engineering and Technology 4(24): 5469-5473, 2012.

[3] Nazil Perveen, Ishan Bhardwaj and ,Darshan Kumar. "An Overview on Template Matching Methodologies and its Applications".International Journal of Research in Computer and Communication Technology, Vol 2, Issue 10, October- 2013
[4] Preeti Tuli, Kavita Ahuja. "Object Recognition by Template Matching Using Correlations and Phase Angle Method" .International Journal of Advanced Research in Computer and Communication Engineering Vol. 2, Issue 3, March 2013.

[5] Er. Priyanka Jarial, Amanpreet Kaur. "Object Recognition using Template matching with the help of Features extraction method". International Research Journal of Engineering and Technology (IRJET) e-ISSN: 2395 -0056 Volume: 02 Issue: 05 | Aug-2015.

[6] Ms. A.Sakila,Dr. S.Vijayarani. "Template Matching Technique For Searching Words In Document Images". International Journal on Cybernetics \& Informatics (IJCI) Vol. 4, No. 6, December 2015.

[7] Nadir Nourain Dawoud,Josefina Janier,Brahim Belhaouari Samir. "Fast Template Matching Method Based Optimized Sum of Absolute Difference Algorithm for Face Localization".International Journal of Computer Applications (0975 - 8887) Volume 18- No.8, March 2011. 